\title{
Energy Harvesting in a Bodipy-Functionalized Rotaxane
}

\author{
Nisa Yesilgul, ${ }^{\S}$ Ozlem Seven, ${ }^{\ddagger}$ Ruslan Guliyev, ${ }^{\ddagger}$ and Engin U. Akkaya*,, \\ ${ }^{\S}$ Department of Chemistry, Bilkent University, Ankara 06800, Turkey \\ ${ }^{\ddagger}$ UNAM-National Nanotechnology Research Center, Bilkent University, Ankara 06800, Turkey \\ Supporting Information
}

\begin{abstract}
A rotaxane composed of two separate Bodipyfunctionalized units can be synthesized with a high yield. The resulting structure shows a very efficient through-space energy transfer (FRET), acting as an energy funnel. Thus, maximum solar output in the visible region can be collected and converted into red light, which can be transformed efficiently with a finetuned photovoltaic device. The versatility of the synthetic pathway demonstrates the potential utility of rotaxane-based energy harvesting supramolecules assemblies.
\end{abstract}

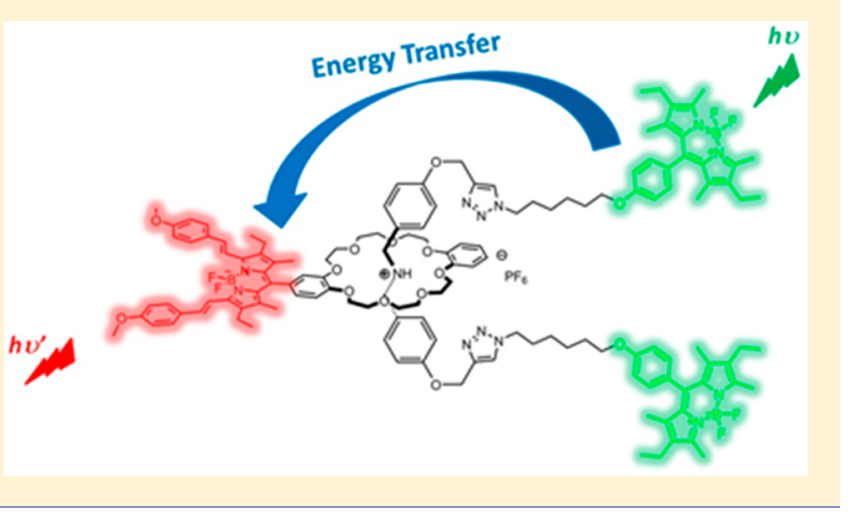

\section{INTRODUCTION}

Organic solar concentrators continue to attract attention. ${ }^{1}$ Dendritic energy funnels with two or more distinct chromophores are an established approach ${ }^{2}$ for obtaining a molecular solar concentrator producing a monochromic emission, which could then be coupled to a high-end photovoltaic device for enhanced efficiency. A promising alternative is to make use of mechanical interlocking, ${ }^{3}$ thus quickly assembling multiple chromophores in close proximity for through -space energy transfer. ${ }^{4}$

Bodipy dyes, on the other hand, proved themselves to be very attractive chromophores in very diverse fields of applications ${ }^{5}$ due to their high photostability and chemical stability coupled with large extinction coefficients in the visible region and impressive quantum yields. Not surprisingly, they attracted attention in various solar cell designs, as well. ${ }^{6}$ Bodipy dyes are also very amenable to modification, ${ }^{7}$ yielding dyes with absorbance peaks covering essentially the entire visible spectrum, and even near IR. Our goal in this work was to assemble a [2] rotaxane making use of dibenzo-fused [24]crown- 8 and dibenzyl ammonium modules. The affinity of this crown unit and the dibenzyl ammonium cation is wellestablished in the literature. ${ }^{8}$

\section{RESULTS AND DISCUSSION}

Our synthesis of the energy funnel rotaxane starts with tosylation of the commercially available oligoethylene glycol $\mathbf{1}$, followed by the closure of the crown ring, yielding formylsubstituted dibenzo-fused 24-crown-8 (3, Scheme 1). Then, meso-substituted Bodipy (4) was synthesized by a wellestablished protocol in Bodipy synthesis. ${ }^{9}$ The next step is the transformation of the green emitting light into a red emitting dye (5) by a reaction with $p$-methoxybenzaldehyde under conditions optimized in our laboratory. ${ }^{10}$ The synthesis of the axle component of the rotaxane starts with $p$ hydroxybenzaldehyde (6), which can easily be reacted with propargyl bromide. Reductive amination using compound 8 in methanol yields dibenzylamine derivative 9 in a high yield. Protonation is followed by ion exchange with $\mathrm{NH}_{4} \mathrm{PF}_{6}$, which yields organic soluble ammonium salt 10. Green emitting absorbing Bodipy modules were synthesized starting from previously reported ${ }^{10 \mathrm{~b}}$ compound $\mathbf{1 1}$; its reaction with sodium azide in DMSO at $100{ }^{\circ} \mathrm{C}$ yields Bodipy compound 12. The final assembly reaction of the rotaxane makes use of the affinity of dibenzylammonium cation for dibenzo-fused [24]-crown-8, which is followed by the click attachment of the chromophore/ stoppers yielding the target supramolecular assembly 13 (Scheme 1 and Figure 1).

In order to assess energy transfer characteristics of the rotaxane, we acquired absorption spectra of the rotaxane and the related modules separately, and as a mixture.

In the absorbance spectrum, the changes are relatively minor (Figure 2). More revealing is the emission spectra of the [2]rotaxane 13 and the modules 5 and 12, separately and as a mixture, Figure 3. The green emission module is highly fluorescent either alone (12) or in the mixture. However, in the mixture, excitation at $500 \mathrm{~nm}$ yields no detectable emission at $675 \mathrm{~nm}$. The energy funneling rotaxane, however, at the same concentrations, yields a very minor peak around $530 \mathrm{~nm}$, while most of the emission is centered around $675 \mathrm{~nm}$ when excited at $500 \mathrm{~nm}$. This is a very clear evidence for energy transfer in rotaxane 13. An excitation spectrum was also acquired in Figure 4. As expected, it shows two peaks when the emission is collected at $673 \mathrm{~nm}$. Energy transfer efficiencies are

Received: August 8, 2018

Published: October 3, 2018 
Scheme 1. Synthesis of the Rotaxane-Based Energy Funnel 13

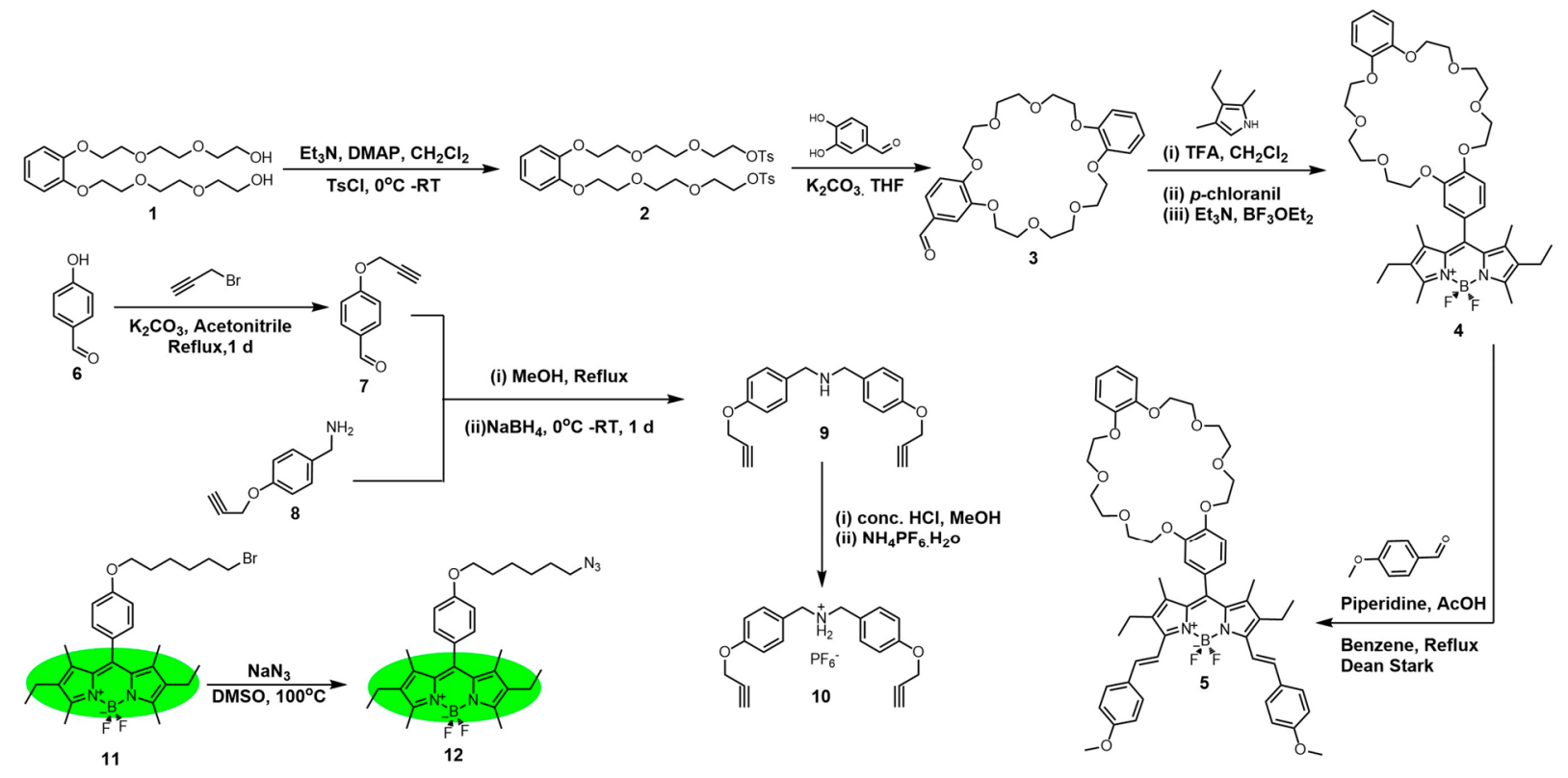

$5+10+12 \underset{\mathrm{CH}_{2} \mathrm{Cl}_{2}, \mathrm{RT}, 1 \mathrm{~d}}{\stackrel{\mathrm{Cu}(\mathrm{MeCN})_{4} \mathrm{PF}_{6}, 2,6-\text { lutidine }}{\longrightarrow}} 13$

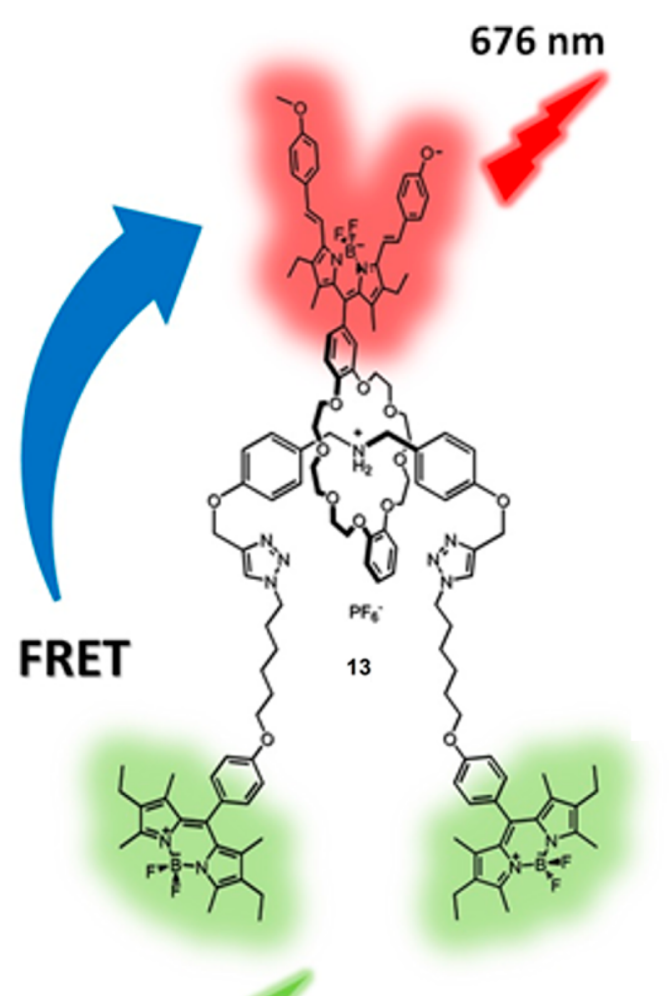

$525 \mathrm{~nm}$

Figure 1. Structure of the target [2]-rotaxane $\mathbf{1 3}$ and the direction of energy transfer and conversion.

often reported with large over estimations ${ }^{11}$ based on the decrease in the quantum yield of the donor chromophore. Thus, a change in the quantum yields of the donor suggests an efficiency of $97 \%$, but a more reliable estimate of energy

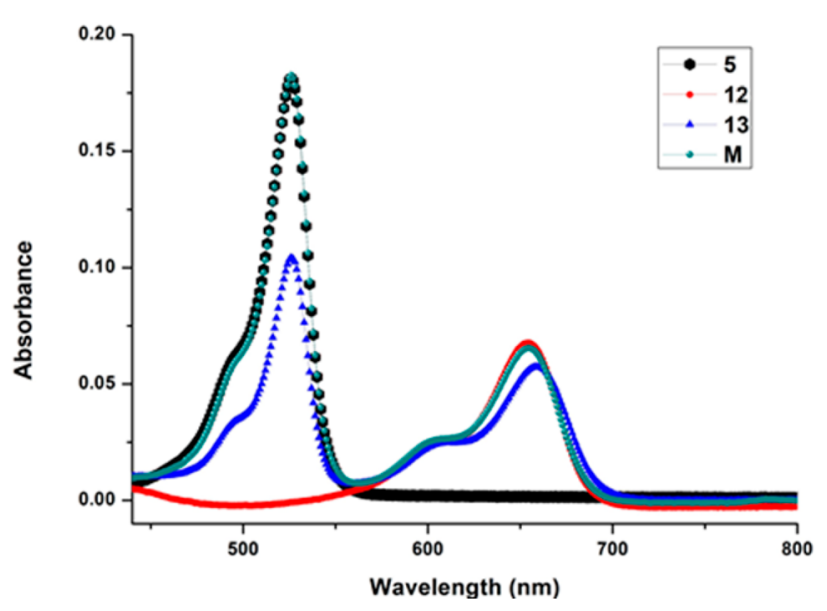

Figure 2. Absorption spectra of [2] rotaxane $13\left(1.0 \times 10^{-6} \mathrm{M}\right)$, compound $5\left(1.0 \times 10^{-6} \mathrm{M}\right)$, compound $12\left(2.0 \times 10^{-6} \mathrm{M}\right)$, and mixture $\mathbf{M}$ (a mixture of 5 and 12 in a molar ratio of $1: 2$ ) in chloroform.

transfer as a function of wavelength can be obtained by the normalizing absorption spectrum and excitation spectrum of the energy transfer cassette, at the peak of the acceptor absorption. ${ }^{12}$ This yields an approximate energy transfer of 40-50\% between 475 and $550 \mathrm{~nm}$.

Modular synthesis of energy-funneling supramolecular systems is likely to find practical applications in organic solar concentrators. In this work, we presented a concise approach for the assembly of a trichromophoric system; however, the idea presented here is fully transferable to a more elaborate multichromophoric assembly, with higher conversion efficiencies. Our work toward that goal is in progress. 


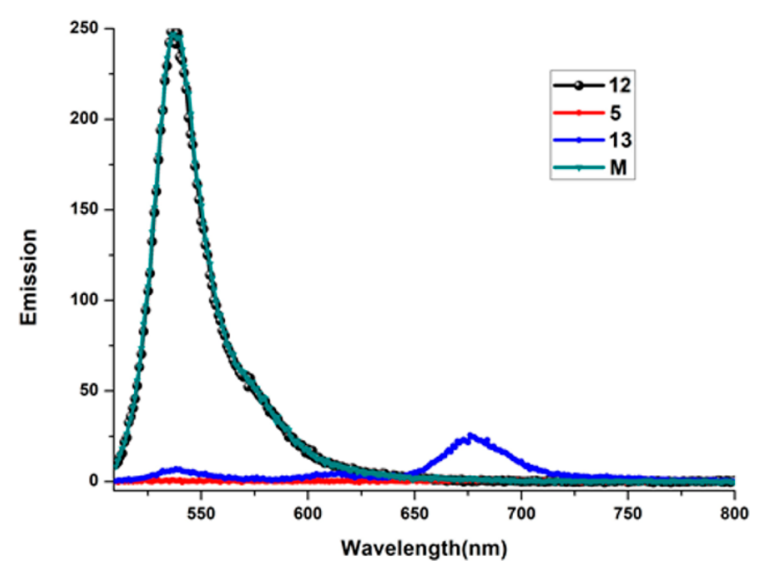

Figure 3. Emission spectra of [2] rotaxane $13\left(1.0 \times 10^{-6} \mathrm{M}\right)$, compound $5\left(1.0 \times 10^{-6} \mathrm{M}\right)$, compound $12\left(2.0 \times 10^{-6} \mathrm{M}\right)$, and mixture $\mathbf{M}$ (a mixture of $\mathbf{5}$ and $\mathbf{1 2}$ in a molar ratio of 1:2) in chloroform. The excitation wavelength of the fluorescent spectra is $500 \mathrm{~nm}$.

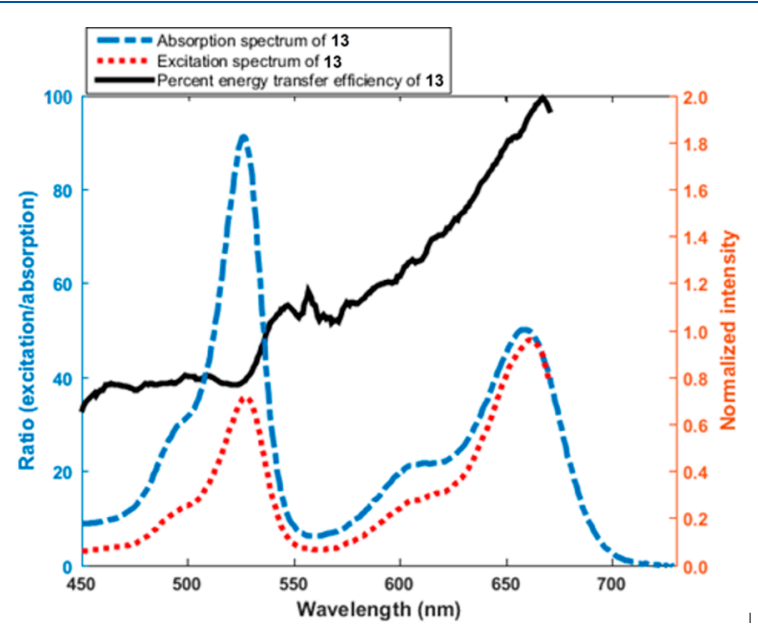

Figure 4. Percent energy transfer efficiency of $\mathbf{1 3}$ (solid line) as a function of wavelength of excitation. Excitation spectrum of 13 (dotted line) and absorption spectrum of 13 (dash-dotted line), normalized at $660 \mathrm{~nm}$. (Emission data were collected at $673 \mathrm{~nm}$.)

\section{EXPERIMENTAL SECTION}

General Procedures. ${ }^{1} \mathrm{H}$ NMR and ${ }^{13} \mathrm{C}$ NMR spectra were recorded on a Bruker DPX-400 (operating at $400 \mathrm{MHz}$ for ${ }^{1} \mathrm{H}$ NMR and $100 \mathrm{MHz}$ for ${ }^{13} \mathrm{C} \mathrm{NMR}$ ) in $\mathrm{CDCl}_{3}$ with tetramethylsilane as an internal standard. All spectra were recorded at $25^{\circ} \mathrm{C}$, and coupling constants ( $J$ values) were given in hertz $(\mathrm{Hz})$. Chemical shifts were given in parts per million ( $\mathrm{ppm})$. Splitting patterns are designated as $\mathrm{s}$ (singlet), $\mathrm{d}$ (doublet), $\mathrm{t}$ (triplet), $\mathrm{q}$ (quartet), $\mathrm{m}$ (multiplet), and $\mathrm{p}$ (pentet). All of the ${ }^{13} \mathrm{C}$ spectra were recorded with simultaneous decoupling of proton nuclei. Melting points were determined with an Electrochemical 9100 apparatus. Mass spectra were recorded on an Agilent Technologies 6530 Accurate-Mass Q-TOF LC/MS system. Absorption spectra were performed by using a Varian Cary- 100 spectrophotometer. Fluorescence measurements were conducted on a Varian Eclipse spectrofluometer. Reactions were monitored by thinlayer chromatography using Merck TLC silica gel $60 \mathrm{~F}_{254}$. Silica gel column chromatography was performed over Merck silica gel 60 (particle size: $0.040-0.063 \mathrm{~mm}, 230-400$ mesh ASTM). All other reagents and solvents were purchased from Aldrich and used without further purification. Compounds $\mathbf{1}^{13}$ and $\mathbf{8}^{8 \mathrm{~b}}$ were synthesized according to the literature.

Synthesis of Compound 2. Compound 1 (5.6 g, $15 \mathrm{mmol})$, triethylamine $(8.7 \mathrm{~mL}, 62 \mathrm{mmol})$, and 4-dimethylamino pyridine (10 $\mathrm{mg}, 0.15 \mathrm{mmol})$ were mixed in DCM $(60 \mathrm{~mL})$ at $0{ }^{\circ} \mathrm{C}$ in an ice bath. 4-Toluenesulfonyl chloride $(7.2 \mathrm{~g}, 38 \mathrm{mmol})$ dissolved in DCM (150 $\mathrm{mL}$ ) was added dropwise to the reaction mixture with vigorous stirring. After the temperature was kept at $0{ }^{\circ} \mathrm{C}$ for $1 \mathrm{~h}$, the ice bath was removed. The reaction mixture was stirred at room temperature overnight. The reaction mixture was washed with $0.1 \mathrm{M} \mathrm{HCl}$ (twice) and saturated $\mathrm{NaCl}$ solutions (twice). The organic layer was dried over $\mathrm{Na}_{2} \mathrm{SO}_{4}$ and concentrated by evaporation. The crude product was purified by column chromatography (silica gel, EtOAc/hexane 1:6 (v/v)). Compound 2 was obtained as a colorless oil $(6.68 \mathrm{~g}, 65 \%$ yield). ${ }^{1} \mathrm{H}$ NMR $\left(400 \mathrm{MHz}, \mathrm{CDCl}_{3}\right): \delta 7.81(\mathrm{~d}, J=8.0 \mathrm{~Hz}, 4 \mathrm{H}), 7.34$ $(\mathrm{d}, J=8.0 \mathrm{~Hz}, 4 \mathrm{H}), 6.93(\mathrm{~s}, 4 \mathrm{H}), 4.18-4.14(\mathrm{q}, J=4.0 \mathrm{~Hz}, 8 \mathrm{H}), 3.84$ $(\mathrm{t}, J=4.0 \mathrm{~Hz}, 4 \mathrm{H}), 3.72-3.68(\mathrm{~m}, 8 \mathrm{H}), 3.64-3.61(\mathrm{~m}, 4 \mathrm{H}), 2.45(\mathrm{~s}$, $6 \mathrm{H}) .{ }^{13} \mathrm{C}$ NMR $\left(100 \mathrm{MHz}, \mathrm{CDCl}_{3}\right): \delta 149.0,144.8,133.1,129.8$, 128.0, 121.7, 115.0, 70.8, 70.8, 69.8, 69.3, 68. 9, 68.7, 21.6 ppm. HRMS (ESI-TOF) $m / z:[\mathrm{M}+\mathrm{Na}]^{+}$calcd for $\mathrm{C}_{32} \mathrm{H}_{42} \mathrm{O}_{12} \mathrm{~S}_{2} \mathrm{Na}$, 705.2010; found, 705.1977.

Synthesis of Compound 3. Under an argon atmosphere, 3,4dihydroxybenzaldehyde $(1.38 \mathrm{~g}, 10 \mathrm{mmol})$ and $\mathrm{K}_{2} \mathrm{CO}_{3}(16.3 \mathrm{~g}, 50$ mmol) were mixed in THF $(300 \mathrm{~mL})$. The mixture was heated under reflux for $1 \mathrm{~h}$, and then compound $2(6.83 \mathrm{~g}, 10 \mathrm{mmol})$ in THF (100 $\mathrm{mL}$ ) was added. The reaction mixture was heated under reflux for 24 h. After the reaction cooled to room temperature, the solvent was removed by evaporation. The residue was dissolved in DCM (200 $\mathrm{mL}$ ) and washed with $1 \mathrm{M} \mathrm{HCl}$ and saturated $\mathrm{NaCl}$ aqueous solutions. The organic layer was dried over $\mathrm{Na}_{2} \mathrm{SO}_{4}$ and concentrated by evaporation. The crude product was purified by column chromatography (silica gel, EtOAC/MeOH 10:1). Compound 3 was obtained as an off-white solid (2.88 g, 60\% yield). Mp: 95.0-97.0 ${ }^{\circ} \mathrm{C}$. ${ }^{1} \mathrm{H}$ NMR $\left(400 \mathrm{MHz}, \mathrm{CDCl}_{3}\right): \delta 9.77(\mathrm{~s}, 1 \mathrm{H}), 7.40-7.33(\mathrm{~m}$, $2 \mathrm{H}), 6.92-6.82(\mathrm{~m}, 5 \mathrm{H}), 4.19-4.11(\mathrm{~m}, 8 \mathrm{H}), 3.92-3.78(\mathrm{~m}, 16 \mathrm{H})$. ${ }^{13} \mathrm{C}$ NMR $\left(100 \mathrm{MHz}, \mathrm{CDCl}_{3}\right): \delta 190.8,154.3,149.2,148.9,148.9$, 130.2, 126.7, 121.4, 121.4, 114.1, 112.0, 111.2, 71.5, 71.4, 71.3, 69.9, 69.7, 69.5, 69.4, 69.4, 69.3 ppm. HRMS (ESI-TOF) $m / z:[\mathrm{M}+\mathrm{Na}]^{+}$ calcd for $\mathrm{C}_{25} \mathrm{H}_{32} \mathrm{O}_{9} \mathrm{Na}$, 499.1939; found, 499.1922.

Synthesis of Dibenzocrown-Substituted Bodipy 4. $\mathrm{CH}_{2} \mathrm{Cl}_{2}(300$ $\mathrm{mL}$ ) was purged with argon for $30 \mathrm{~min}$. Compound $3(500 \mathrm{mg}, 1.04$ $\mathrm{mmol})$ and 3-ethyl-2,4-dimethyl pyrrole $(0.33 \mathrm{~mL}, 2.41 \mathrm{mmol})$ were added. The color of the solution turned to red after the addition of 2 drops of trifluoroacetic acid. The reaction mixture was stirred at room temperature overnight. Then, $p$-chloranil $(283 \mathrm{mg}, 1.15 \mathrm{mmol})$ was added, and the reaction mixture was stirred at room temperature for 2 h. Then triethyl amine $(1.3 \mathrm{~mL})$ and boron trifluoride diethyl etherate $(1.3 \mathrm{~mL})$ were added sequentially. After the mixture was stirred at room temperature for $30 \mathrm{~min}$, the reaction mixture was extracted with water. The organic layer was dried over $\mathrm{Na}_{2} \mathrm{SO}_{4}$ and concentrated by evaporation. The crude product was purified by column chromatography (silica gel, EtOAC/hexane 2:1 (v/v)). Compound 4 was obtained as a red wax $(0.33 \mathrm{~g}, 44 \%$ yield $) .{ }^{1} \mathrm{H}$ NMR $(400 \mathrm{MHz}$, $\left.\mathrm{CDCl}_{3}\right): \delta 6.98-6.90(\mathrm{~m}, 5 \mathrm{H}), 6.82(\mathrm{~d}, J=8.0 \mathrm{~Hz}, 2 \mathrm{H}), 4.24-4.17$ $(\mathrm{m}, 6 \mathrm{H}), 4.15-4.11(\mathrm{~m}, 2 \mathrm{H}), 3.87-4.02(\mathrm{~m}, 16 \mathrm{H}), 2.54(\mathrm{~s}, 6 \mathrm{H}), 2.32$ $(\mathrm{q}, J=8.0 \mathrm{~Hz}, 4 \mathrm{H}), 1.38(\mathrm{~s}, 6 \mathrm{H}), 1.00(\mathrm{t}, J=8.0 \mathrm{~Hz}, 6 \mathrm{H}) .{ }^{13} \mathrm{C} \mathrm{NMR}$ $\left(100 \mathrm{MHz}, \mathrm{CDCl}_{3}\right): \delta 153.6,149.6,149.3,149.0,139.9,138.4,132.7$, 131.0, 128.5, 121.5, 121.4, 114.2, 114.1, 113.9, 71.5, 71.4, 71.3, 69.98, 69.91, 69.87, 69.6, 69.5, 69.4, 69.3, 17.1, 14.6, 12.5, 11.8 ppm. HRMS (ESI-TOF) $m / z:[\mathrm{M}+\mathrm{Na}]^{+}$calcd for $\mathrm{C}_{41} \mathrm{H}_{53} \mathrm{BF}_{2} \mathrm{~N}_{2} \mathrm{O}_{8} \mathrm{Na}$, 772.3792; found, 772.3793 .

Extended-Conjugation Chromophore 5. Compound $4(259 \mathrm{mg}$ $0.345 \mathrm{mmol}$ ) and 4-methoxy benzaldehyde $(105 \mu \mathrm{L}, 0.862 \mathrm{mmol})$ were dissolved in benzene $(40 \mathrm{~mL})$. Piperidine $(0.32 \mathrm{~mL})$ and acetic acid $(0.32 \mathrm{~mL})$ were added to the reaction mixture. The reaction mixture was refluxed using a Dean-Stark apparatus until all of the aldehyde was consumed. After the reaction was completed, it was extracted with DCM and water. The organic layer was dried over $\mathrm{Na}_{2} \mathrm{SO}_{4}$ and concentrated by evaporation. The crude product was purified by silica gel column chromatography (first DCM/MeOH 95:5 then EtOAC/hexane 2:1 (v/v)). Compound 5 was obtained as a green solid (0.18 g, 54\% yield). Mp: $212.2-214.1{ }^{\circ} \mathrm{C}$ (decomp). ${ }^{1} \mathrm{H}$ $\operatorname{NMR}\left(400 \mathrm{MHz}, \mathrm{CDCl}_{3}\right): \delta 7.68(\mathrm{~d}, J=16.8 \mathrm{~Hz}, 2 \mathrm{H}), 7.59(\mathrm{~d}, J=$ $7.6 \mathrm{~Hz}, 4 \mathrm{H}), 7.23(\mathrm{~d}, J=16.0 \mathrm{~Hz}, 2 \mathrm{H}), 7.01-7.91(\mathrm{~m}, 9 \mathrm{H}), 6.85(\mathrm{~d}, J$ 
$=10.0 \mathrm{~Hz}, 2 \mathrm{H}), 4.29-4.12(\mathrm{~m}, 10 \mathrm{H}), 4.01-3.91(\mathrm{~m}, 14 \mathrm{H}), 3.88(\mathrm{~s}$, $6 \mathrm{H}), 4.02(\mathrm{q}, J=8.0 \mathrm{~Hz}, 4 \mathrm{H}), 1.43(\mathrm{~s}, 6 \mathrm{H}), 1.18(\mathrm{t}, J=8.0 \mathrm{~Hz}, 6 \mathrm{H})$. ${ }^{13} \mathrm{C}$ NMR $\left(100 \mathrm{MHz}, \mathrm{CDCl}_{3}\right): \delta 160.2,150.4,149.6,149.4,138.8$, $137.7,135.3,133.5,130.4,128.8,121.5,121.5,118.2,114.2,71.5,71.4$, $71.3,71.3,70.0,69.9,69.9,69.6,69.5,69.4,69.4,55.4,29.7,18.4$, 14.0, $11.6 \mathrm{ppm}$. HRMS (ESI-TOF) $\mathrm{m} / z:[\mathrm{M}+\mathrm{K}]^{+}$calcd for $\mathrm{C}_{57} \mathrm{H}_{65} \mathrm{BF}_{2} \mathrm{~N}_{2} \mathrm{O}_{10} \mathrm{~K}$, 1024.4368; found, 1024.4389 .

Synthesis of 4-Propargyloxybenzaldehyde 7. To a solution of $\mathrm{K}_{2} \mathrm{CO}_{3}(1.50 \mathrm{~g}, 7.3 \mathrm{mmol})$ in acetonitrile $(100 \mathrm{~mL})$ were added 4hydroxybenzaldehyde $(0.1 \mathrm{~g}, 0.82 \mathrm{mmol})$ and propargyl bromide $(0.11 \mathrm{~g}, 0.90 \mathrm{mmol})$, and the mixture was refluxed for 2 days under an argon atmosphere. Then, the reaction mixture was cooled and concentrated under reduced pressure. The residue was dissolved in $\mathrm{CH}_{2} \mathrm{Cl}_{2}(100 \mathrm{~mL})$, filtrated, and then washed with water $(100 \mathrm{~mL})$ three times. The organic phase was dried with $\mathrm{Na}_{2} \mathrm{SO}_{4}$ and concentrated under reduced pressure. The crude product was purified by column chromatography (eluent $\mathrm{CHCl}_{3}$ ) to afford a white solid (0.98 g, 74\% yield). Mp: 82.0-84.0 ${ }^{\circ} \mathrm{C} .{ }^{1} \mathrm{H}$ NMR (400 MHz, $\left.\mathrm{CDCl}_{3}\right): \delta 9.90(\mathrm{~s}, 1 \mathrm{H}), 7.85(\mathrm{~d}, J=8.5 \mathrm{~Hz}, 2 \mathrm{H}), 7.09(\mathrm{~d}, J=8.5 \mathrm{~Hz}$, $2 \mathrm{H}), 4.78$ (s, $2 \mathrm{H}), 2.59$ (s, $1 \mathrm{H}) .{ }^{13} \mathrm{C}$ NMR $\left(100 \mathrm{MHz}, \mathrm{CDCl}_{3}\right): \delta$ $190.6,162.4,132.3,130.59,115.16,77.58,76.40,56.11$. HRMS (ESITOF) $m / z:[\mathrm{M}+\mathrm{H}]^{+}$calcd for $\mathrm{C}_{10} \mathrm{H}_{9} \mathrm{O}_{2}, 161.0597$; found, 161.0569.

Synthesis of the Dibenzylamine Compound 9. Compound 7 $(0.43 \mathrm{~g}, 2.67 \mathrm{mmol})$ and compound $8(0.43 \mathrm{~g}, 2.67 \mathrm{mmol})$ were mixed in methanol $(20 \mathrm{~mL})$, and the mixture was refluxed for $24 \mathrm{~h}$. Then, the reaction mixture was cooled to $0{ }^{\circ} \mathrm{C}$, and $\mathrm{NaBH}_{4}(1.0 \mathrm{~g}$, $26.4 \mathrm{mmol}$ ) was added portionwise. The reaction mixture was stirred at room temperature for $24 \mathrm{~h}$. Water was added to the reaction. and the mixture was concentrated under vacuum pressure. The residue was dissolved in $\mathrm{CH}_{2} \mathrm{Cl}_{2}(100 \mathrm{~mL})$ and was washed with water (100 $\mathrm{mL}$ ) three times. The organic phase was dried with $\mathrm{Na}_{2} \mathrm{SO}_{4}$ and concentrated under reduced pressure. The crude product was purified by column chromatography (eluent 100:1 DCM/MeOH) to afford a yellow oil $\left(0.50 \mathrm{~g}, 61 \%\right.$ yield). ${ }^{1} \mathrm{H}$ NMR $\left(400 \mathrm{MHz}, \mathrm{CDCl}_{3}\right): \delta 7.30$ $(\mathrm{d}, J=8.4 \mathrm{~Hz}, 4 \mathrm{H}), 6.97(\mathrm{~d}, J=8.5 \mathrm{~Hz}, 4 \mathrm{H}), 4.69(\mathrm{~d}, J=2.3 \mathrm{~Hz}, 4 \mathrm{H})$, $3.76(\mathrm{~s}, 4 \mathrm{H}), 2.56(\mathrm{t}, J=2.3 \mathrm{~Hz}, 2 \mathrm{H}) .{ }^{13} \mathrm{C} \mathrm{NMR}\left(100 \mathrm{MHz}, \mathrm{CDCl}_{3}\right)$ : $\delta 156.7,133.4,129.4,114.9,78.8,75.65,55.9,52.4$. HRMS (ESITOF) $m / z:[\mathrm{M}+\mathrm{H}]^{+}$calcd for $\mathrm{C}_{20} \mathrm{H}_{20} \mathrm{NO}_{2}, 306.1489$; found, 306.1517.

Synthesis of Compound 10. Compound 9 ( $0.50 \mathrm{~g}, 1.61 \mathrm{mmol})$ was dissolved in methanol $(15 \mathrm{~mL})$, and concentrated $\mathrm{HCl}$ was added to adjust the $\mathrm{pH}$ lower than 2; then, the solvent was removed in vacuo. The reaction residue was dissolved in acetone $(15 \mathrm{~mL})$, and a saturated solution of $\mathrm{NH}_{4} \mathrm{PF}_{6}$ was added dropwise until the reaction mixture became clear. The solvent was removed under reduced pressure, and water was added to the residue. The resulting mixture was filtered, and the residue was washed with water several times and dried to give a white solid $(0.68 \mathrm{~g}, 94 \%$ yield $)$. $\mathrm{Mp}: 238.0-240.0^{\circ} \mathrm{C}$. ${ }^{1} \mathrm{H}$ NMR (400 MHz, MeOD): $\delta 7.44(\mathrm{~d}, J=8.7 \mathrm{~Hz}, 4 \mathrm{H}), 7.07(\mathrm{~d}, J=$ $8.8 \mathrm{~Hz}, 4 \mathrm{H}), 4.77(\mathrm{~d}, J=2.4 \mathrm{~Hz}, 4 \mathrm{H}), 4.18(\mathrm{~s}, 4 \mathrm{H}), 2.95(\mathrm{t}, J=2.4$ $\mathrm{Hz}, 2 \mathrm{H}) .{ }^{13} \mathrm{C}$ NMR (100 MHz, MeOD): $\delta 158.6,131.2,123.7,115.2$, 78.1, 75.7, 55.3, 50.0. HRMS (ESI-TOF) $\mathrm{m} / z$ : $\left[\mathrm{M}-\mathrm{PF}_{6}\right]^{+}$calcd for $\mathrm{C}_{20} \mathrm{H}_{20} \mathrm{NO}_{2}$, 306.1494; found, 306.1510.

Synthesis of Compound 12. Compound $11(0.40 \mathrm{~g}, 0.72 \mathrm{mmol})$ and $\mathrm{NaN}_{3}(0.12 \mathrm{~g}, 1.79 \mathrm{mmol})$ were dissolved in DMSO $(20 \mathrm{~mL})$, and the reaction mixture was heated to $100{ }^{\circ} \mathrm{C}$ for $2 \mathrm{~h}$. The reaction was controlled by TLC. When the reaction was complete, it was cooled to room temperature and $\mathrm{CHCl}_{3}(100 \mathrm{~mL})$ was added and washed with water $(100 \mathrm{~mL})$ six times. The organic layer was dried with $\mathrm{Na}_{2} \mathrm{SO}_{4}$ and concentrated under reduced pressure. The crude product was used without further purification. A dark red solid (0.35 g, $97 \%$ yield) was afforded. Mp: $102.0-103.0{ }^{\circ} \mathrm{C}$ (decomp). ${ }^{1} \mathrm{H}$ NMR $\left(400 \mathrm{MHz}, \mathrm{CDCl}_{3}\right): \delta 7.17(\mathrm{~d}, J=8.4 \mathrm{~Hz}, 2 \mathrm{H}), 7.01(\mathrm{~d}, J=8.4 \mathrm{~Hz}$, $2 \mathrm{H}), 4.04(\mathrm{t}, J=6.4 \mathrm{~Hz}, 2 \mathrm{H}), 3.32(\mathrm{t}, J=6.8 \mathrm{~Hz}, 2 \mathrm{H}), 2.55(\mathrm{~s}, 6 \mathrm{H})$, $2.32(\mathrm{q}, J=7.5 \mathrm{~Hz}, 4 \mathrm{H}), 1.94-1.80(\mathrm{~m}, 2 \mathrm{H}), 1.71-1.64(\mathrm{~m}, 2 \mathrm{H})$, $1.61-1.49(\mathrm{~m}, 4 \mathrm{H}), 1.36(\mathrm{~s}, 6 \mathrm{H}), 1.00(\mathrm{t}, J=7.5 \mathrm{~Hz}, 6 \mathrm{H}) .{ }^{13} \mathrm{C} \mathrm{NMR}$ $\left(100 \mathrm{MHz}, \mathrm{CDCl}_{3}\right): \delta 159.5,153.5,140.4,138.4,132.6,131.2,129.4$, $127.8,115.0,67.9,51.4,29.1,28.8,26.6,25.7,17.1,14.6,12.5,11.8$. HRMS (ESI-TOF) $m / z:[\mathrm{M}+\mathrm{Na}]^{+}$calcd for $\mathrm{C}_{29} \mathrm{H}_{38} \mathrm{BF}_{2} \mathrm{~N}_{5} \mathrm{ONa}$, 543.3066; found, 543.3039.
Synthesis of Rotaxane 13. A solution of compound 5 (326 mg, $0.33 \mathrm{mmol})$ and compound $10(100 \mathrm{mg}, 0.22 \mathrm{mmol})$ was dissolved in degassed DCM $(15 \mathrm{~mL})$ and stirred at room temperature for $4 \mathrm{~h}$. Then, compound $12(252 \mathrm{mg}, 0.48 \mathrm{mmol})$ in $5 \mathrm{~mL}$ of DCM and $\mathrm{Cu}\left(\mathrm{CH}_{3} \mathrm{CN}\right)_{4} \mathrm{PF}_{6}(74 \mathrm{mg}, 0.20 \mathrm{mmol})$ and 2,6-lutidine $(5 \mu \mathrm{L}, 0.107$ $\mathrm{mmol})$ were added. The resulting mixture was stirred at room temperature for 1 day. After 1 day, DCM $(25 \mathrm{~mL})$ was added to the reaction mixture and it was washed with water $(30 \mathrm{~mL})$. The organic layer was dried with $\mathrm{Na}_{2} \mathrm{SO}_{4}$ and concentrated under reduced pressure. The crude product was purified with column chromatography over silica gel (9:1 DCM/MeOH). Compound 13 was afforded as a dark purple solid (175 mg, 32\% yield). Mp: $204.0-206.0{ }^{\circ} \mathrm{C}$

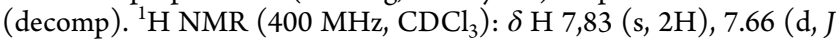
$=16.8 \mathrm{~Hz}, 2 \mathrm{H}), 7.58(\mathrm{~d}, J=8.8 \mathrm{~Hz}, 4 \mathrm{H}), 7.38(J=1.2 \mathrm{~Hz}, 4 \mathrm{H}), 7.25$ $(\mathrm{d}, J=8.0 \mathrm{~Hz}, 2 \mathrm{H}), 7.15(\mathrm{~d}, J=8.4 \mathrm{~Hz}, 4 \mathrm{H}), 7.05(\mathrm{~d}, J=8.0 \mathrm{~Hz}, 2 \mathrm{H})$, $7(\mathrm{~s}, 2 \mathrm{H}), 6.97(\mathrm{~d}, J=4.8 \mathrm{~Hz}, 4 \mathrm{H}), 6.94(\mathrm{~d}, J=4.8 \mathrm{~Hz}, 4 \mathrm{H}), 6.90(\mathrm{~d}, J$ $=8.0 \mathrm{~Hz}, 4 \mathrm{H}), 6.82(\mathrm{~s}, 1 \mathrm{H}), 6.78-6.73(\mathrm{~m}, 2 \mathrm{H}), 5.19(\mathrm{~s}, 4 \mathrm{H}) 4.50$ $(\mathrm{m}, 2 \mathrm{H}), 4.41(\mathrm{t}, J=7.2 \mathrm{~Hz}), 4.31-4.26(\mathrm{~m}, 2 \mathrm{H}), 4.19-4.16(\mathrm{~m}$, $2 \mathrm{H}), 4.11-4.10(\mathrm{~m} 2 \mathrm{H}), 4.05-4.04(\mathrm{~m}, 2 \mathrm{H}), 4.01(\mathrm{t}, J=6.4 \mathrm{~Hz}$, $4 \mathrm{H})$, 3.95-3.93 (m, 2H), 3.90-3.88 (m, 2H), $3.87(\mathrm{~s}, 6 \mathrm{H}$, ArOCH3), 3.73-3.69 (m, 2H), 3.67-364 (m, 2H), 3.52-3.42 (m, 6H), $3.37-3.34(\mathrm{~m}, 2 \mathrm{H}), 2.54(\mathrm{~s}, 12 \mathrm{H}), 2.31\left(\mathrm{q}, J_{1}=7.2 \mathrm{~Hz}, J_{2}=7.6 \mathrm{~Hz}\right.$, $10 \mathrm{H}), 2.04-1.95(\mathrm{~m}, 6 \mathrm{H}), 1.88-1.80(\mathrm{~m}, 6 \mathrm{H}), 1.47-1.44(\mathrm{~m}, 4 \mathrm{H})$, $1.35(\mathrm{~s}, 12 \mathrm{H}), 1.25(\mathrm{~s}, 6 \mathrm{H}), 1.13(\mathrm{t}, J=7.2 \mathrm{~Hz}, 6 \mathrm{H}), 1.00(\mathrm{t}, J=7.2$ $\mathrm{Hz}, 12 \mathrm{H}) .{ }^{13} \mathrm{C}$ NMR $\left(100 \mathrm{MHz}, \mathrm{CDCl}_{3}\right): \delta 160.3,159.5,159.0$, $153.4,150.6,148.5,148.4,147.2,143.2,140.4,138.5$, 138.3, 135.6, $133.7,132.6,130.7,130.2,129.4,128.8,128.3,127.7,123.9,123.5$, $121.9,118.0,115.1,114.9,114.3,70.7,67.8,61.6,55.4,52.01,50.3$, $30.2,29.7,29.7,29.1,26.3,25.6,22.7,18.4,17.1,14.6,14.0,12.49$, $12.46,12.44,11.9,11.4$. HRMS (ESI-TOF) $\mathrm{m} / z:\left[\mathrm{M}-\mathrm{PF}_{6}\right]^{+}$calcd for $\mathrm{C}_{135} \mathrm{H}_{161} \mathrm{~B}_{3} \mathrm{~F}_{6} \mathrm{~N}_{13} \mathrm{O}_{14}, 2333.2651$; found, 2333.2310.

\section{ASSOCIATED CONTENT}

\section{S Supporting Information}

The Supporting Information is available free of charge on the ACS Publications website at DOI: 10.1021/acs.joc.8b01928.

Spectral data and copies of ${ }^{1} \mathrm{H}$ and ${ }^{13} \mathrm{C}$ spectra for new compounds (PDF)

\section{AUTHOR INFORMATION}

\section{Corresponding Author}

*E-mail: eua@fen.bilkent.edu.tr.

ORCID

Engin U. Akkaya: 0000-0003-4720-7554

Notes

The authors declare no competing financial interest.

\section{ACKNOWLEDGMENTS}

We gratefully acknowledge support from Bilkent University.

\section{REFERENCES}

(1) Chemisana, D. Building Integrated Concentrating Photovoltaics. Renewable Sustainable Energy Rev. 2011, 15, 603-611.

(2) Mirloup, A.; Retailleau, P.; Ziessel, R. Luminescent molecular solar concentrators made of multi-Bodipy dyes. Tetrahedron Lett. 2013, 54, 4456-4462.

(3) (a) Saha, S.; Stoddart, J. F. Photo-driven molecular devices. Chem. Soc. Rev. 2007, 36, 77-92. (b) Crowley, J. D.; Leigh, D. A.; Lusby, P. J.; McBurney, R. T.; Perret-Aebi, L.-E.; Petzold, C.; Slawin, A. M. Z.; Symes, M. D. A. A Switchable Palladium-Complexed Molecular Shuttle and Its Metastable Positional Isomers. J. Am. Chem. Soc. 2007, 129, 15085-15090. (c) Harada, A.; Hashidzume, A.; Yamaguchi, H.; Takashima, Y. Polymeric Rotaxanes. Chem. Rev. 2009, 109, 5974-6073. (d) Zhang, Z.-J.; Zhang, Y.-M.; Liu, Y. Controlled Molecular Self-Assembly Behaviors between Cucurbituril and Bispyridinium Derivatives. J. Org. Chem. 2011, 76, 4682-4685. 
(e) Kobr, L.; Zhao, K.; Shen, Y.; Comotti, A.; Bracco, S.; Shoemaker, R. K.; Sozzani, P.; Clark, N. A.; Price, J. C.; Rogers, C. T.; Michl, J. Inclusion Compound Based Approach to Arrays of Artificial Dipolar Molecular Rotors. A Surface Inclusion. J. Am. Chem. Soc. 2012, 134, 10122-10131. (f) Gallina, M. E.; Baytekin, B.; Schalley, C.; Ceroni, P. Light-harvesting in multichromophoric rotaxanes. Chem. - Eur. J. 2012, 18, 1528-1535. (g) Arumugaperumal, R.; Srinivasadesikan, V.; Raju, M. V. R.; Lin, M.-C.; Shukla, T.; Singh, R.; Lin, H.-C. Acid/Base and $\mathrm{H}_{2} \mathrm{PO}_{4}{ }^{-}$Controllable High-Contrast Optical Molecular Switches with a Novel BODIPY Functionalized [2]Rotaxane. ACS Appl. Mater. Interfaces 2015, 7, 26491-26503.

(4) (a) Devadoss, C.; Bharathi, P.; Moore, J. S. Energy Transfer in Dendritic Macromolecules: Molecular Size Effects and the Role of an Energy Gradient. J. Am. Chem. Soc. 1996, 118, 9635-9644. (b) Hiratani, K.; Kaneyama, M.; Nagawa, Y.; Koyama, E.; Kanesato, M. Synthesis of [1]Rotaxane via Covalent Bond Formation and Its Unique Fluorescent Response by Energy Transfer in the Presence of Lithium Ion. J. Am. Chem. Soc. 2004, 126, 13568-13569. (c) Onagi, H.; Rebek, J., Jr. Fluorescence resonance energy transfer across a mechanical bond of a rotaxane. Chem. Commun. 2005, 4604-4606.

(5) (a) Ziessel, R.; Ulrich, G.; Haefele, A.; Harriman, A. An Artificial Light-Harvesting Array Constructed from Multiple Bodipy Dyes. J. Am. Chem. Soc. 2013, 135, 11330-11344. (b) Wang, J.; Wu, Q.; Wang, S.; Yu, C.; Li, J.; Hao, E.; Wei, Y.; Mu, X.; Jiao, L. Conformation-Restricted Partially and Fully Fused BODIPY Dimers as Highly Stable Near-Infrared Fluorescent Dyes. Org. Lett. 2015, 17, $5360-5363$

(6) (a) Liao, J.; Zhao, H.; Xu, Y.; Zhou, W.; Peng, F.; Wang, Y.; Fang, Y. Novel BODIPY dyes with electron donor variety for dyesensitized solar cells. RSC Adv. 2017, 7, 33975-33985. (b) Klfout, H.; Stewart, A.; Elkhalifa, M.; He, H. BODIPYs for Dye-Sensitized Solar Cells. ACS Appl. Mater. Interfaces 2017, 9, 39873-39889.

(7) (a) Killoran, J.; Allen, L.; Gallagher, J. F.; Gallagher, W. M.; O'Shea, D. F. Synthesis of $\mathrm{BF}_{2}$ chelates of tetraarylazadipyrromethenes and evidence for their photodynamic therapeutic behaviour. Chem. Commun. 2002, 1862-1863. (b) Jiao, L.; Yu, C.; Uppal, T.; Liu, M.; Li, Y.; Zhou, Y.; Hao, E.; Hu, X.; Vicente, M. G. H. Long wavelength red fluorescent dyes from 3,5-diiodo-BODIPYs. Org. Biomol. Chem. 2010, 8, 2517-2519. (c) Isaad, J.; El Achari, A. A water soluble fluorescent BODIPY dye with azathia-crown ether functionality for mercury chemosensing in environmental media. Analyst 2013, 138, 3809-3819. (d) Salim, M. M.; Owens, E. A.; Gao, T.; Lee, J. H.; Hyun, H.; Choi, H. S.; Henary, M. Hydroxylated near-infrared BODIPY fluorophores as intracellular pH sensors. Analyst 2014, 139, 4862-4873. (e) Kilic, B.; Yesilgul, N.; Polat, V.; Gercek, Z.; Akkaya, E. U. Bodipy-based photosensitizers with long alkyl tails at the meso position: efficient singlet oxygen generation in Cremophor-EL micelles. Tetrahedron Lett. 2016, 57, 1317-1320.

(8) (a) Ashton, P. R.; Bartsch, R. A.; Cantrill, S. J.; Hanes, R. E.; Hickingbottom, S. K.; Lowe, J. N.; Preece, J. A.; Stoddart, J. F.; Talanov, V. S.; Wang, Z. H. Secondary dibenzylammonium ion binding by $[24]$ crown- 8 and $[25]$ crown- 8 macrocycle. Tetrahedron Lett. 1999, 40, 3661-3664. (b) Zhang, Z. J.; Zhang, H. Y.; Wang, H.; Liu, Y. A Twin-Axial Hetero[7]rotaxane. Angew. Chem., Int. Ed. 2011, 50, 10834-10838.

(9) (a) Ulrich, G.; Ziessel, R.; Harriman, A. The Chemistry of Fluorescent Bodipy Dyes: Versatility Unsurpassed. Angew. Chem., Int. Ed. 2008, 47, 1184-1201. (b) Bozdemir, Ö. A.; Büyükcakir, O.; Akkaya, E. U. Novel Molecular Building Blocks Based on the Boradiazaindacene Chromophore: Applications in Fluorescent Metallosupramolecular Coordination Polymers. Chem. - Eur. J. 2009, 15, $3830-3838$

(10) (a) Buyukcakir, O.; Bozdemir, O. A.; Kolemen, S.; Erbas, S.; Akkaya, E. U. Tetrastyryl-Bodipy Dyes: Convenient Synthesis and Characterization of Elusive Near IR Fluorophores. Org. Lett. 2009, 11, 4644-4647. (b) Kostereli, Z.; Ozdemir, T.; Buyukcakir, O.; Akkaya, E. U. Tetrastyryl-BODIPY-Based Dendritic Light Harvester and Estimation of Energy Transfer Efficiency. Org. Lett. 2012, 14, 36363639.
(11) (a) Hawker, C. J.; Frechet, J. M. J. Preparation of polymers with controlled molecular architecture. A new convergent approach to dendritic macromolecules. J. Am. Chem. Soc. 1990, 112, 7638-7647. (b) Hawker, C. J.; Frechet, J. M. J. A new convergent approach to monodisperse dendritic macromolecules. J. Chem. Soc., Chem. Commun. 1990, 1010-1013. (c) Li, F.; Yang, S. I.; Ciringh, Y.; Seth, J.; Martin, C. H.; Singh, D. L.; Kim, D.; Birge, R. R.; Bocian, D. F.; Holten, D.; Lindsey, J. L. Design, Synthesis, and Photodynamics of Light-Harvesting Arrays Comprised of a Porphyrin and One, Two, or Eight Boron-Dipyrrin Accessory Pigments. J. Am. Chem. Soc. 1998, 120, 10001-10017. (d) Adronov, A.; Frechet, J. M. J. Lightharvesting dendrimers. Chem. Commun. 2000, 1701-1710. (e) Dichtel, W. R.; Hecht, S.; Frechet, J. M. J. Functionally Layered Dendrimers: A New Building Block and Its Application to the Synthesis of Multichromophoric Light-Harvesting Systems. Org. Lett. 2005, 7, 4451-4454. (f) Bozdemir, O. A.; Yilmaz, M. D.; Buyukcakir, O.; Siemiarczuk, A.; Tutas, M.; Akkaya, E. U. Convergent synthesis and light harvesting properties of dendritic boradiazaindacene (BODIPY) appended perylenediimide dyes. New J. Chem. 2010, 34, 151-155. (g) Ziessel, R.; Harriman, A. Artificial light-harvesting antennae: electronic energy transfer by way of molecular funnels. Chem. Commun. 2011, 47, 611-631.

(12) Roberts, D. V.; Wittmershaus, B. P.; Zhang, Y.-Z.; Swan, S.; Klinosky, M. P. Efficient excitation energy transfer among multiple dyes in polystyrene microspheres. J. Lumin. 1998, 79, 225-231.

(13) Liu, D.; Wang, D.; Wang, M.; Zheng, Y.; Koynov, K.; Auernhammer, G. K.; Butt, H.-J.; Ikeda, T. Supramolecular Organogel Based on Crown Ether and Secondary Ammoniumion Functionalized Glycidyl Triazole Polymers. Macromolecules 2013, 46, 4617-4625. 